\title{
An analysis of influenza outbreaks in institutions and enclosed societies
}

\author{
T. J. R. FINNIE*, V. R. COPLEY, I. M. HALL AND S. LEACH \\ Microbial Risk Assessment and Behavioural Science, Emergency Response Department, Public Health England, \\ Porton Down, UK
}

Received 26 July 2012; Final revision 11 February 2013; Accepted 4 March 2013; first published online 10 April 2013

\section{SUMMARY}

This paper considers the reported attack ratio arising from outbreaks of influenza in enclosed societies. These societies are isolated from the wider community and have greater opportunities for contact between members which would aid the spread of disease. While the particular kind of society (prison, care home, school, barracks, etc.) was not a significant factor in an adjusted model of attack ratio, a person's occupation within the society was. In particular, children and military personnel suffer a greater attack ratio than other occupational types (staff, prisoners, etc.). There was no temporal trend in final attack ratio nor, with the exception of 1918, do pandemic years show abnormal attack ratios. We also observed that as community size increases, the attack ratio undergoes steep nonlinear decline. This statistical analysis draws attention to how the organization of such societies, their size and the occupations of individuals within them affect the final attack ratio.

Key words: Analysis of data, community epidemics, infectious disease control, influenza, mathematical modelling.

\section{INTRODUCTION}

The transmission of respiratory infectious diseases and, a usual epidemiological corollary of this, the final clinical attack ratio at the end of epidemics is known to be influenced by factors such as population density and the patterns and intensity of population mixing at different spatial and social scales [1-3]. In the case of influenza, secondary attack ratios in households have often been shown to decrease with increasing household size [4] and attack ratios are also generally expected to be higher in schools than in the wider community [5]. Here we use the dimen-

\footnotetext{
* Author for correspondence: T. J. R. Finnie, Microbial Risk Assessment and Behavioural Science, Emergency Response Department, Health Protection Agency, Porton Down, Salisbury, Wiltshire, SP4 0JG, UK.

(Email: Thomas.Finnie@phe.gov.uk)
}

sionless terminology attack 'ratio' to represent the proportion of the population who fall ill with influenza over the whole reported outbreak.

Enclosed societies form an important spatial scale between the household and the general population. These societies are diverse both in terms of their function and the extent to which social interactions are encouraged or allowed between members and subgroups. Some form part of an institution (prisons, schools, the military, etc); others are isolated by natural factors. Individuals within these societies may be sufficiently close that repeated contact with the same people each day may enhance the mechanisms for disease transmission. In certain societies the importation of disease may have a significant impact on the members of the society. Following importation, diseases that are subclinical or difficult to differentiate on clinical grounds may spread quickly before local 
Table 1. Descriptive statistics of available risk factors for an enclosed society attack ratio

\begin{tabular}{|c|c|c|c|c|}
\hline Characteristic & $\begin{array}{l}\text { Number of } \\
\text { institutional } \\
\text { groups }(n=83)\end{array}$ & $\begin{array}{l}\text { Number of } \\
\text { individuals } \\
(n=39602)\end{array}$ & $\begin{array}{l}\text { Attack } \\
\text { ratio }(\%)\end{array}$ & $\begin{array}{l}\text { Median } \\
(\mathrm{IQR})\end{array}$ \\
\hline \multicolumn{5}{|l|}{ Year } \\
\hline 1890 & 37 & 8121 & 20 & \\
\hline 1918 & 10 & 9353 & 32 & \\
\hline 1920 & 1 & 1000 & 44 & \\
\hline 1957 & 3 & 223 & 73 & \\
\hline 1972 & 2 & 4148 & 22 & \\
\hline 1976 & 1 & 859 & 24 & \\
\hline 1978 & 2 & 1107 & 52 & \\
\hline 1980 & 1 & 859 & 23 & \\
\hline 1983 & 1 & 859 & 29 & \\
\hline 1984 & 1 & 555 & 14 & \\
\hline 1986 & 1 & 859 & 21 & \\
\hline 1996 & 2 & 1804 & 44 & \\
\hline 1997 & 5 & 211 & 40 & \\
\hline 2000 & 2 & 41 & 22 & \\
\hline 2001 & 2 & 80 & 31 & \\
\hline 2002 & 1 & 3000 & 3 & \\
\hline 2004 & 2 & 1481 & 17 & \\
\hline 2009 & 9 & 5042 & 17 & \\
\hline \multicolumn{5}{|l|}{ Institution category } \\
\hline School & 24 & 9115 & 36 & \\
\hline Barracks & 3 & 8239 & 18 & \\
\hline Children's home & 9 & 1366 & 27 & \\
\hline Hospital and care home & 19 & 4330 & 16 & \\
\hline Isolated community & 1 & 3200 & 18 & \\
\hline Prison & 18 & 9428 & 13 & \\
\hline Ship & 9 & 3924 & 53 & \\
\hline \multicolumn{5}{|l|}{ Occupation type } \\
\hline Children & 30 & 14120 & 32 & \\
\hline Military personnel & 9 & 11560 & 28 & \\
\hline Patients and residents & 12 & 3279 & 15 & \\
\hline Prisoners & 13 & 9084 & 13 & \\
\hline Staff & 19 & 1559 & 19 & \\
\hline Group size & & & & $185(49-610)$ \\
\hline
\end{tabular}

IQR, Interquartile range.

behaviour is changed to mitigate the outbreak [6-10]. Furthermore, some of these societies may contain members that are particularly susceptible to infection or are severely compromised by infection (immunocompromised people).

The aim of this study is twofold, first, to analyse the reported outbreaks of influenza in enclosed societies over the past 120 years to determine the quantitative evidence for the hypotheses that attack ratios can be higher in certain types of enclosed societies than others; and second, to test the further hypothesis that in enclosed societies with larger populations there is a tendency for lower final attack ratios. It was recognized that there would be confounding factors to such an analysis, as, for example, the strain of influenza implicated in the outbreak and the past history of influenza in a community. These factors are unreported in most published data.

\section{METHODS}

\section{Data}

The data collected and described by Finnie et al. [11] provide information on influenza outbreaks in 43 enclosed institutions over the years 1890-2009 (Table 1). The outbreaks are distinguished by the 
Table 2. Estimates of fixed effects for an enclosed society attack ratio. All regression models have year and institution as random effects. Referent is the subgroup of a categorical variable against which other subgroups are compared in the regression mode

\begin{tabular}{|c|c|c|c|c|}
\hline Characteristic & $\begin{array}{l}\text { Unadjusted OR } \\
(95 \% \mathrm{CI})\end{array}$ & $P$ value & $\begin{array}{l}\text { Adjusted OR } \\
(95 \% \mathrm{CI})\end{array}$ & $P$ value \\
\hline \multicolumn{5}{|l|}{ Institution category } \\
\hline School & Referent & & Referent & \\
\hline Barracks & $0 \cdot 17(0 \cdot 05-0 \cdot 59)$ & & $0 \cdot 67(0 \cdot 12-3 \cdot 73)$ & \\
\hline Children's home & $0.55(0 \cdot 17-1 \cdot 77)$ & & $0.58(0.20-1.65)$ & \\
\hline Hospital and care home & $0 \cdot 34(0 \cdot 14-0 \cdot 86)$ & & $2 \cdot 18(0 \cdot 83-5 \cdot 73)$ & \\
\hline Isolated community & $0 \cdot 38(0 \cdot 06-2 \cdot 55)$ & & $1 \cdot 49(0 \cdot 27-8 \cdot 24)$ & \\
\hline Prison & $0 \cdot 24(0 \cdot 11-0 \cdot 55)$ & & $1.06(0.44-2 \cdot 57)$ & \\
\hline Ship & $0 \cdot 89(0 \cdot 39-2 \cdot 01)$ & 0.02 & $1 \cdot 44(0 \cdot 44-4 \cdot 70)$ & $0 \cdot 40$ \\
\hline \multicolumn{5}{|l|}{ Occupation type } \\
\hline Children & Referent & & Referent & \\
\hline Military personnel & $0 \cdot 56(0 \cdot 25-1 \cdot 29)$ & & $0 \cdot 73(0 \cdot 19-2 \cdot 86)$ & \\
\hline Patients and residents & $0 \cdot 36(0 \cdot 23-0 \cdot 54)$ & & $0 \cdot 12(0 \cdot 07-0 \cdot 20)$ & \\
\hline Prisoners & $0.35(0 \cdot 22-0 \cdot 54)$ & & $0.26(0.16-0.44)$ & \\
\hline Staff & $0 \cdot 47(0 \cdot 33-0 \cdot 68)$ & $<0 \cdot 01$ & $0 \cdot 16(0 \cdot 10-0 \cdot 25)$ & $<0.01$ \\
\hline Group size (ln) & $0 \cdot 80(0 \cdot 75-0 \cdot 86)$ & $<0 \cdot 01$ & $0.63(0.57-0.69)$ & $<0 \cdot 01$ \\
\hline
\end{tabular}

OR, Odds ratio; CI, confidence interval.

occupational group of the institution's population affected and the size of this population, as well as the final number of individuals who acquired influenza. The data summarize 9737 cases of influenza occurring in a total population of about 39600 .

Institutions with regimens that permit more frequent, longer term and more 'intimate' contacts between individuals would generally be expected to promote increased overall attack ratios. The contact regimen may be related to the purpose of an institution and institutions were accordingly classified into one of seven categories on the basis of their function (Table 1). In addition, as, a priori, we would expect there to be differences in attack ratios due to different activities, so each of the population groups within an institution was categorized into a broad occupational type (Table 1).

\section{Statistical analysis}

Attack ratios are correlated within years and institutions and we accordingly fitted mixed-effects logistic regression models [12]. Year and institution were modelled as normally distributed random effects with a mean of zero. Year was effectively a proxy for influenza strain. Pandemic years might be expected to have larger outbreaks due to larger susceptible groups in wider populations and so the normality assumption for year was tested using the AndersonDarling test (a standard test for a sample of data being derived from a normally distributed population) [13]. Institution was a proxy for local response and other similar factors. Group size, occupational type and institution category were included as fixed effects, at first in separate models and then in a joint multiple regression to obtain adjusted estimates of their effects. Occupation type was to some extent confounded with institution category and we were not able to examine the interaction between these two effects due to data limitations. Group size follows a right-skewed distribution and was allowed to vary on a natural logarithmic scale in the model as initial investigation revealed this provided a better fit to the data than a linear term. Attack ratio data were converted to a binomial response variable and analysed with the lme4 package [14] in R using a logit link function. The significance of the random and fixed effects was assessed using likelihood ratio tests.

Table 1 shows that data are included for one isolated community consisting of 3200 individuals. Data on occupational groups within this population were not available and so for the purposes of statistical analysis the entire community population was assigned to the 'children' occupation category. The sensitivity of results to this assignment were investigated by omitting the community from this category 


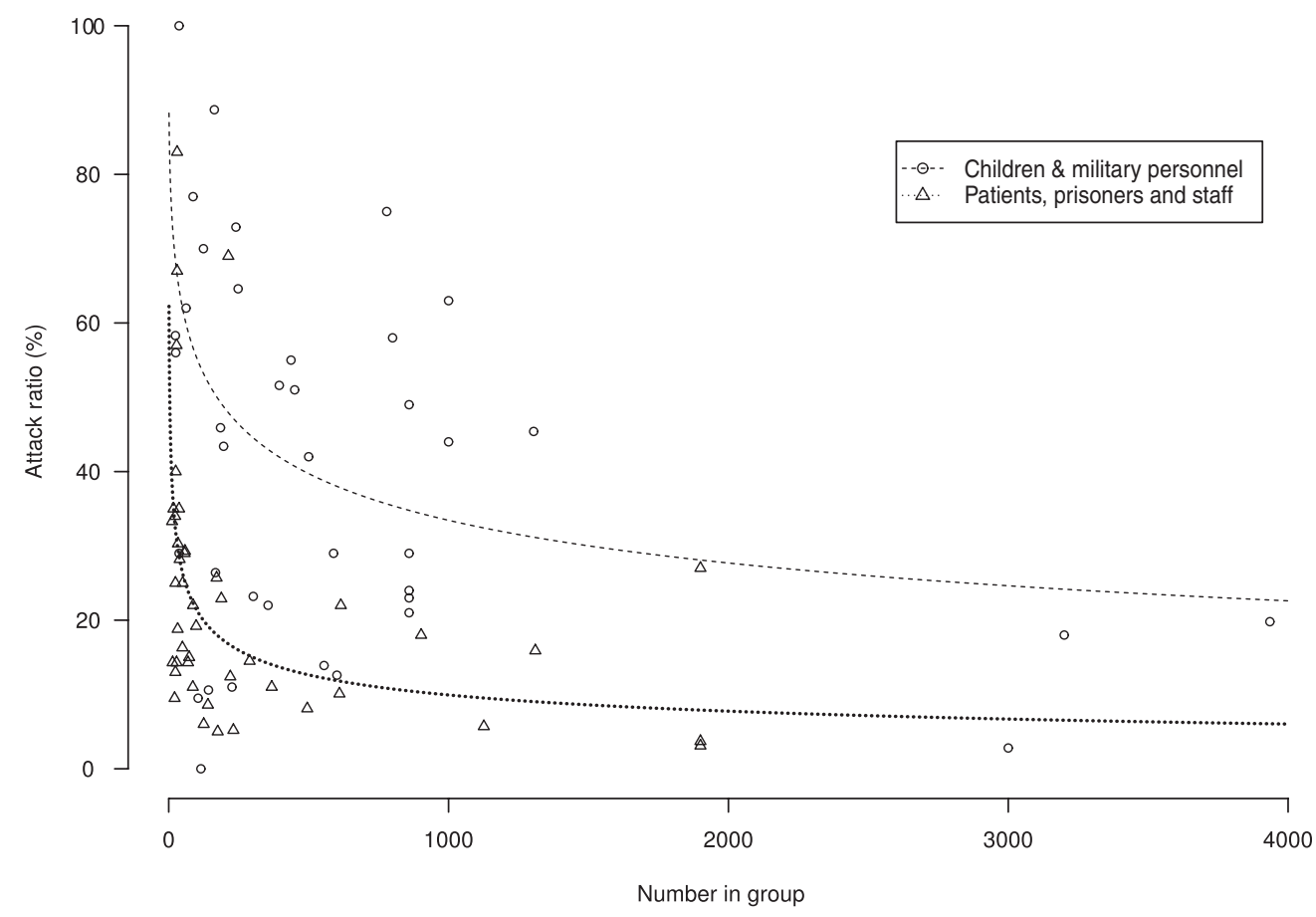

Fig. 1. Attack ratio and group size by broad occupational type. Estimates of effect sizes drawn from multiple regression models using mean value of zero for random effects, two occupational categories as shown, and reference institution category (school).

and placing it in an occupational category of its own, for the single fixed-effect regression, or omitting it from the analysis altogether for the multiple regression. Resultant changes in model coefficients and significance levels were then examined.

\section{RESULTS}

Unadjusted estimates from the single covariate mixed effects regressions of institution category, occupation category and group size on attack ratio are given in the second column of Table 2. Adjusted estimates of these effects from the multiple regression are given in the fourth column of Table 2. Although all fixed effects are significant at the 5\% level when considered singly, only occupation category and group size remain significant at the $5 \%$ level in the multiple regression. The random effects of year and institution were significant at the 5\% level in all models. The normal distribution was found to be a reasonable approximation to the year random effect as the Anderson-Darling test did not reject the hypothesis of normality at the $5 \%$ level.

The unadjusted odds ratios show that the odds of suffering from influenza in an enclosed institution are on average lower for individuals in barracks, children's homes, hospitals, isolated communities, prisons and ships than they are for individuals in schools $(P=0.02) \quad$ (Table 2). The estimates for barracks (OR 0·17, 95\% CI 0.05-0.59), hospitals and care homes (OR 0.34, 95\% CI 0·14-0.86) and prisons (OR $0 \cdot 24,95 \%$ CI $0 \cdot 11-0 \cdot 55$ ) are significantly different from the baseline estimate for schools. Occupation type within an enclosed institution is, however, more significant than institution category per se as an unadjusted fixed effect $(P<0 \cdot 01)$. The odds of acquiring influenza are on average much lower for patients and residents (OR $0 \cdot 36,95 \%$ CI $0 \cdot 23-0 \cdot 54)$, prisoners (OR $0 \cdot 35,95 \%$ CI $0 \cdot 22-0 \cdot 68$ ) and staff (OR 0.47, 95\% CI $0 \cdot 33-0.68)$ than they are for children. These estimates are not sensitive to the occupation type assigned to the isolated community as the estimates of effect size vary by an average of only $4 \%$ between the alternative assignments (log scale). The unadjusted effect of group size also indicates that the odds of acquiring influenza decrease by $20 \%$ for every one unit increase in the log of group size (OR $0 \cdot 80,95 \%$ CI $0 \cdot 75-0 \cdot 86$ ).

Occupational type and group size remain significant in the multiple regression model of attack ratios $(P<0 \cdot 01)$ but institutional category is not significant $(P=0 \cdot 40)$. Patients and residents in enclosed 


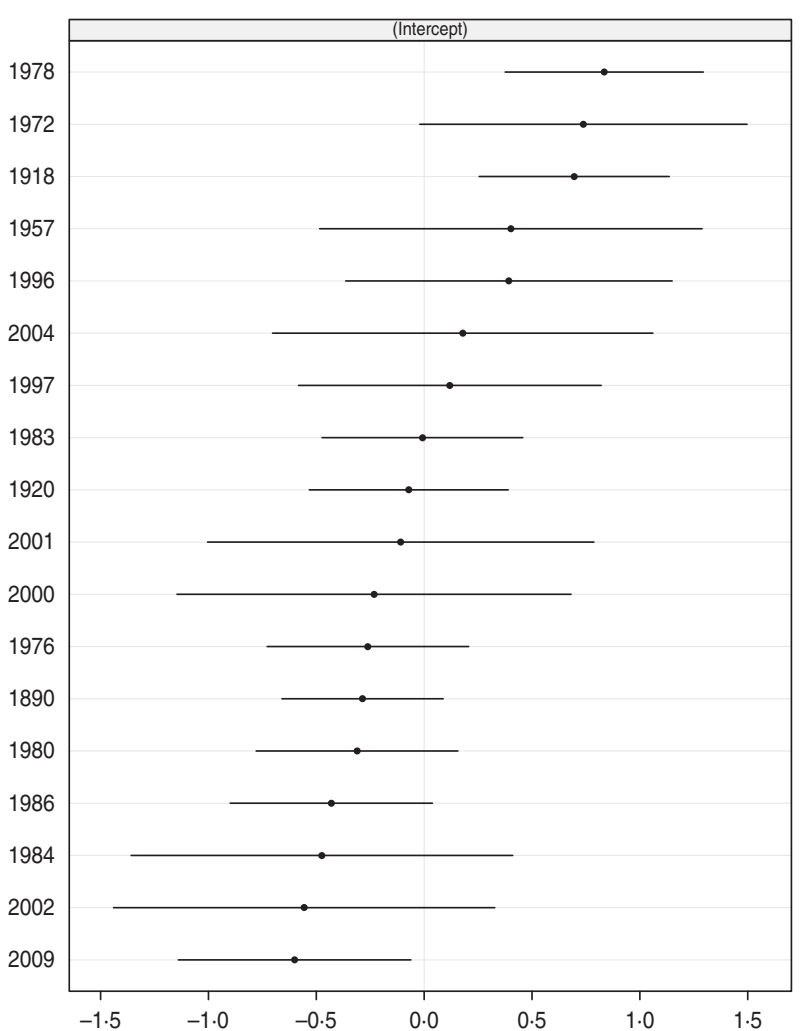

Fig. 2. Magnitude, direction and confidence of effect of year, modelled as a random variable, on the attack ratio. Estimates taken from an adjusted model. Points show best estimate of the effect with lines showing the 95\% confidence intervals. Years where the effect is positive had a greater attack ratio than would be expected given the other modelled effects, while those that are negative had a smaller attack ratio than would be expected.

institutions have odds of acquiring influenza on average only $12 \%$ of those of a child in an enclosed institution (adjusted OR 0.12, 95\% CI 0.07-0.20). Similar odds ratios are seen for prisoners (adjusted OR 0.26, 95\% CI $0 \cdot 16-0 \cdot 44)$ and staff (OR $0 \cdot 16,95 \%$ CI $0 \cdot 1-$ $0 \cdot 25)$. These estimates are not sensitive to the inclusion of the isolated community observation as the coefficient estimates varied by an average of only $0 \cdot 1 \%$ between the observation omitted model and the model in which isolated community was allocated to the 'children' occupational type. The odds ratio for group size in the adjusted model falls to 0.63 (95\% CI 0.57-0.69), indicating that after adjustment for occupation and institution category the odds of acquiring influenza in an enclosed institution decrease by $37 \%$ for every one unit increase in the log of group size (OR 0.63, 95\% CI 0.57-0.69). The combined effects of group size and occupational type are demonstrated in Figure 1 which gives the estimated expected decrease in attack ratio associated with an increase in community size for two broad categories of occupational type. These two categories were created by amalgamating those occupational types with statistically similar effect sizes (statistical characteristics). Figure 1 shows that for a given group size the occupational types of children and military personnel are on average associated with higher attack ratios than the occupational types of patients, prisoners, and staff. Figure 1 also illustrates that as community size increases, the attack ratio undergoes a steep nonlinear decline.

Estimates of the random effects obtained for year and institution are given in Figures 2 and 3, respectively. Year acts as a proxy for both the strain of, and local susceptibility to, the influenza virus and the methods of outbreak control deployed. Pandemic years might a priori be expected to have higher attack ratios due to higher susceptibility but we found no clear temporal trend in the random effect observed by year (Fig. 2). The institution random effect will reflect the general nature of the institution's regimen, including local response to outbreaks. Groups within more relaxed regimens suffer greater attack ratios given similar institution type, occupation type and size (Fig. 3).

\section{DISCUSSION}

Review of past outbreaks illustrates the difficulty in determining the attack ratio of influenza in enclosed societies. Each outbreak is essentially the result of a series of random events marked by the choices and contacts made by the people involved, any prior immunity, the response of public health officials and the susceptibility and size of the population into which the disease is brought. In order to fully understand the enhanced attack ratios in context would require contemporary measures of the wider community attack ratio, which is very difficult to source due to the nature of reporting behaviour.

Notwithstanding these difficulties, from historical data we have examined the hypotheses that attack ratios can be higher in certain types of enclosed societies than others, and that larger populations in enclosed societies are associated with lower final attack ratios. Our results indicate it is occupation type within an institution, rather than institutional category per se, that is a significant driver of final attack ratio. We also found that attack ratio decreases as enclosed community size increases. 


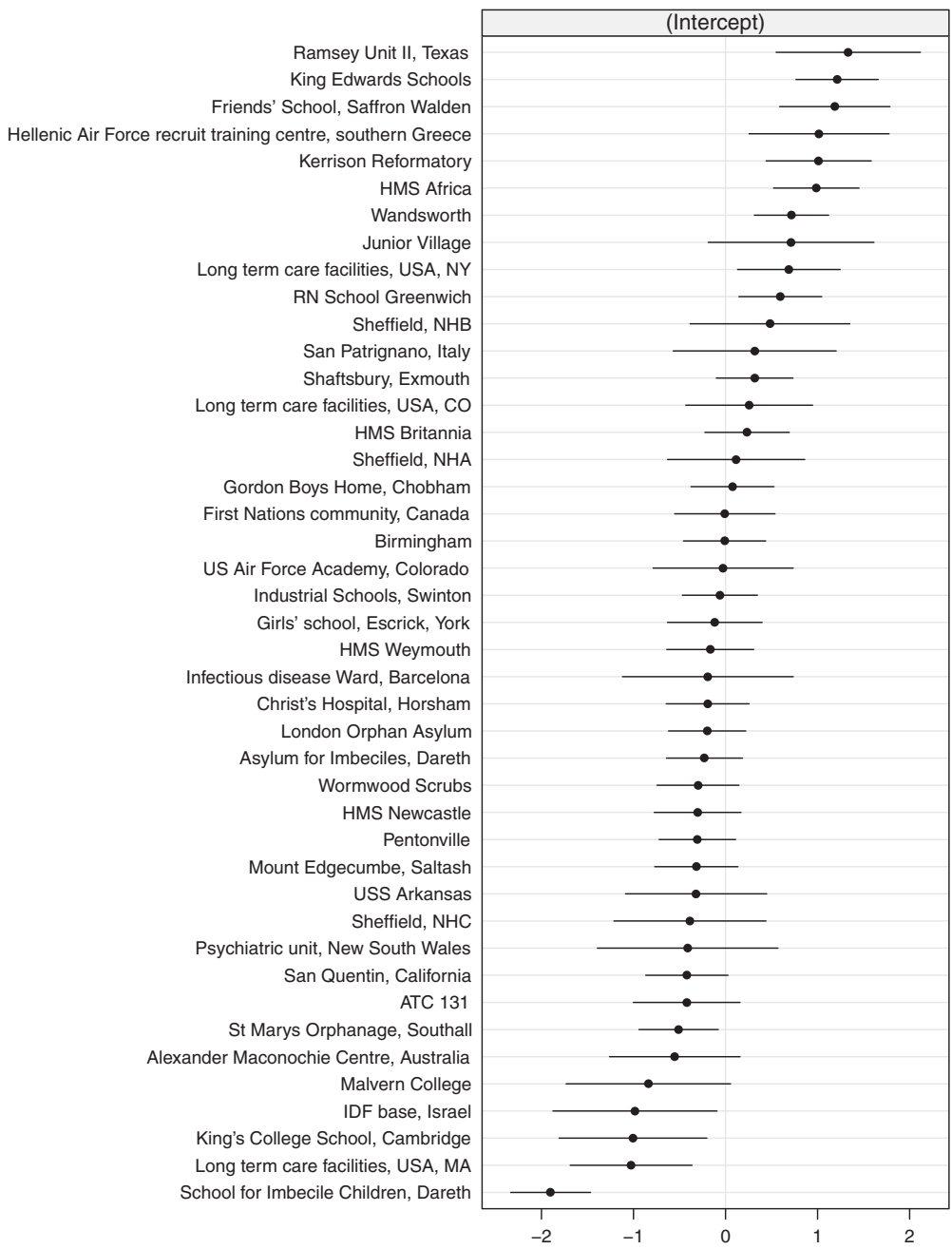

Fig. 3. Magnitude, direction and confidence of effect of individual institution on the attack ratio modelled as a random variable. Estimates taken from an adjusted model. Points show best estimate of the effect with lines showing the 95\% confidence intervals. Institutions where the effect is positive had a greater attack ratio than would be expected given the other modelled effects, while those that are negative had a smaller attack ratio than would be expected.

The significance of occupational type as a predictor of attack ratio may be seen as a reflection of the 'strictness' of the regimen across various institutions. Children and military personnel form an occupational type that is subject to fewer restrictions and/or exhibits more mixing on average than the occupational type categories of patients, prisoners and staff. The latter three categories have a lower attack ratio, on average, than the former, visualized as the difference between the lines in Figure 1. The effects of community size and regimen strictness are different facets of the same underlying process, the ability of people within the community to make contacts and have interactions which spread the disease. Additionally, larger communities may have logistical challenges in diagnosis of members that are suffering illness, particularly if they are self-reporting.
We found no clear trend over time in the random effect observed by year (Fig. 2). Attack ratio would not necessarily be expected to reflect advances in medical technology, unless antiviral counter-measures were in widespread use during the outbreaks. Had mortality ratio been used then a declining trend could have been expected but there were insufficient mortality data to perform this analysis.

The particular nature of an institution's regimen also has a large part to play in determining the final attack ratio. Ceteris paribus, institutions where there is greater interaction have many times the attack ratio of similar organizations which are more restrictive in their mixing patterns. For example the largest, positive, random effect for institution of 1.33 (Fig. 3) gives a point estimate for attack ratio of $79 \%$ for an observation in the reference categories with median 
group size of 185 and a value of zero for the year random effect. The same observation with the minimum estimated random effect for institution of -1.9 has a point estimate for attack ratio of only $13 \%$, a difference in attack ratio potentially caused by an institutional regimen of $66 \%$.

The reports analysed here of influenza in enclosed societies show two clear trends in attack ratio. The first is as the size of a community increases, the attack ratio decreases. This is likely to be caused by scale effects within the community. In the extreme case of a very large community we would expect the attack ratio to approach that of the general population whereas in these small communities it can be significantly greater. The second trend is that attack ratio depends more on the occupation of the individuals within a community than on the type of community itself, so children and soldiers are at greater risk of attack than those in other reported occupations even within the same society. This is likely to be an effect of the differing nature of the contacts these groups make. Overlaid on these broad trends is the influence of the regimen of a society. Where broadly similar societies are attacked those with regimens that are more restrictive in allowing contacts are likely to suffer fewer infections than those that are more open.

Perhaps surprisingly there was no discernible temporal trend in the random effects for year in which an outbreak took place nor were there markedly higher random effects for pandemic years, with the exception of 1918. Pandemic years are spread through the range of attack rates. Attacks in 1918 appear significantly worse but otherwise, attacks in enclosed societies during pandemics are no worse than at other times. There are more reports from pandemic years and probably more societies are attacked but inside an enclosed society the attack ratio for a pandemic strain seems no different to other strains. This might be anticipated as an enclosed society may well be as naive to a seasonal strain, due to rare importations, as society as a whole is to pandemic strains. More detailed analysis of this effect requires measures of the attack ratios both within the enclosed society and the wider community. In the future, serological evidence of random cross-sections of the population may be compared to serological evidence from local outbreaks [15]. The collection and reporting of this information would assist in quantifying both relative attack ratios and the risk of disease importation to these enclosed societies.

\section{ACKNOWLEDGEMENTS}

This work was supported by the European Commission Seventh Framework Programme FluModCont project and Department of Health HPA Grant-in-Aid funding. The views expressed in this article are those of the authors and not necessarily those of the European Commission, DG Research, Health Protection Agency or the Department of Health.

\section{DECLARATION OF INTEREST}

None.

\section{REFERENCES}

1. Gani R, Leach $\mathbf{S}$. Transmission potential of smallpox in contemporary populations. Nature 2001; 414: 748-751.

2. Weiss RA, McMichael AJ. Social and environmental risk factors in the emergence of infectious diseases. Nature Medicine 2004; 10: S70-S76.

3. Ferguson NM, et al. Strategies for mitigating an influenza pandemic. Nature 2006; 442: 448-452.

4. Viboud $\mathbf{C}$, et al. Risk factors of influenza transmission in households. International Congress Series 2004; 1263: 291-294.

5. Cauchemez S, et al. Closure of schools during an influenza pandemic. Lancet Infectious Diseases 2009; 9: 473-481.

6. Ministry of Health. The Pandemic of Influenza 1918-19. London: HMSO, 1920.

7. Parsons HF. Influenza Epidemic of 1889-90. London: HMSO, 1891.

8. Earhart KC, et al. Outbreak of influenza in highly vaccinated crew of U.S. Navy ship. Emerging Infectious Diseases 2001; 7: 463-465.

9. Makras P, et al. Outbreak of meningococcal disease after an influenza B epidemic at a Hellenic air force recruit training center. Clinical Infectious Diseases 2001; 33: e48-e 50.

10. Awofeso N, et al. Influenza outbreak in a correctional facility. Australian and New Zealand Journal of Public Health 2001; 25: 443-446.

11. Finnie TJR, et al. Behaviour and control of influenza in institutions and small societies. Journal of the Royal Society of Medicine 2012; 105: 66-73.

12. Pinheiro JC. Mixed-Effects Models in $S$ and S-PLUS New York: Springer, 2000.

13. Stephens MA. Tests based on edf statistics. In: D'Agostino RB, Stephens MA, eds. Goodness of Fit Techniques. New York: Dekker, 1986, pp. 97-194.

14. Bates D, et al. The lme4 Package (http://cran. r-project.org/web/packages/lme4/index.html). Accessed 10 March 2011.

15. Miller E, et al. Incidence of 2009 pandemic influenza A $\mathrm{H} 1 \mathrm{~N} 1$ infection in England: a cross-sectional serological study. Lancet 2010; 375: 1100-1108. 